\title{
Uterine Corpus Cancer
}

National Cancer Institute

\section{Source}

National Cancer Institute. Uterine Corpus Cancer. NCI Thesaurus. Code C61574.

A malignant neoplasm arising from the uterine corpus. This category includes

endometrial carcinoma and carcinosarcoma. 